\title{
Prognostic impact of preoperative serum interleukin-6 levels in patients with early-stage oral squamous cell carcinoma, defined by sentinel node biopsy
}

\author{
HIROYUKI GODA $^{1}$, MASATO OKAMOTO ${ }^{2}$, KOH-ICHI NAKASHIRO ${ }^{1}$, \\ SATOSHI HINO $^{1}$, RYUICHI MURASE ${ }^{1}$ and HIROYUKI HAMAKAWA ${ }^{1}$ \\ ${ }^{1}$ Department of Oral and Maxillofacial Surgery, Ehime University Graduate School of Medicine, Tōon, Ehime 791-0295; \\ ${ }^{2}$ Department of Advanced Immunotherapeutics, Kitasato University School of Pharmacy, Tokyo 108-8641, Japan
}

Received September 9, 2015; Accepted February 12, 2017

DOI: $10.3892 / \mathrm{ol} .2017 .7183$

\begin{abstract}
Failure to detect recurrence and lymph node metastasis early represents a fundamental barrier to the improvement of survival rate in early stage oral squamous cell carcinoma (OSCC). The present study evaluated the association between serum interleukin-6 (IL-6) level and clinical outcomes in patients with early stage OSCC patients defined by sentinel node biopsy (SNB). A total of 53 patients with clinical stage I/II OSCC who underwent SNB were enrolled. SNB was determined by a radioisotope method, and was evaluated by histopathological examination and genetic analysis. Preoperative sera were measured for IL- 6 by ELISA. In the clinical stage I/II patients, disease-free survival (DFS) was demonstrated to be higher in patients with negative SNB compared with patients with positive SNB. In total, 13 patients were demonstrated to exhibit lymph node metastasis by SNB or were reclassified to pathological stage $\mathrm{T} 4$ subsequent to analysis of the surgically resected specimens. Thus, 40 patients were diagnosed with early stage OSCC. Of these 40 patients, DFS of the patients with low serum IL-6 was significantly higher compared with the patients with high serum IL-6 $(\mathrm{P}=0.012)$. In 19 patients with negative SNB and low serum IL-6, the disease-free rate was $100 \%$. These findings suggested that SNB staging and serum IL-6 level have a high prognostic value in patients with early stage OSCC. Additional investigation and longer follow-up times are warranted to improve understanding of the group of patients that may benefit from this procedure.
\end{abstract}

Correspondence to: Dr Hiroyuki Goda, Department of Oral and Maxillofacial Surgery, Ehime University Graduate School of Medicine, 454 Shitsukawa, Tōon, Ehime 791-0295, Japan

E-mail: hiro9832@m.ehime-u.ac.jp

Key words: sentinel node biopsy, interleukin-6, oral squamous cell carcinoma, biomarker

\section{Introduction}

Oral squamous cell carcinoma (OSCC), the most common type of head and neck carcinoma, represents the fifth most frequently occurring cancer worldwide (1). An estimated 263,900 new cases and 128,000 mortalities occurred globally in 2008 (2). Despite advances in surgery, radiotherapy and chemotherapy, little improvement in the relative survival has been observed in OSCC during the past 30 years (3).

Early-stage OSCC (clinical stage I or II) (4) is primarily managed with surgery. The nodal status of the cervical lymph nodes remains an important prognostic factor in OSCC (5-6). The presence of cervical lymph node metastasis reduces the survival of patients with SCC of the upper aerodigestive tract by up to $50 \%$ (7). Therefore, early detection of cervical lymph node metastasis is hypothesized to improve survival. However, the diagnostic accuracy of lymph node metastasis using imaging tools including ultrasonography (US), computed tomography (CT), magnetic resonance imaging (MRI) and positron emission tomography (PET) is $\sim 70 \%$ (8). Furthermore, patients with delayed neck node metastases generally exhibit a poor prognosis (9).

Sentinel node biopsy (SNB) has been demonstrated to be an oncologically safe staging modality in patients with early stage OSCC, allowing for an individualized and minimally invasive treatment of the neck, and significantly affecting tumor control and survival (10).

However, patients with tumor recurrence and metastases generally exhibit poor prognosis, and predictive biomarkers that identify the risk of tumor relapse may become a powerful tool for follow-up and development of effective treatment plans for these patients (11-13). Sera derived from patients with early stage OSCC were previously examined for multiple cytokines using a multiplexed measurement system (14) and serum interleukin-6 (IL-6) level was revealed to negatively correlate with a favourable outcome in these patients. IL-6 is a multifunctional cytokine that functions in the regulation of inflammatory and immune responses. IL-6 is produced by a variety of cells, primarily monocytes, macrophages and several types of tumor cell during infection and immunological challenge (15). Previous studies have revealed that IL-6 is involved in cancer progression, 
including proliferation, angiogenesis and lymphangiogenesis in several types of cancer, including OSCC (16-19), and worsens cancer prognosis (20-22). The aim of the present study was to determine the function of serum IL- 6 concentration in patients with early-stage OSCC defined by SNB.

\section{Materials and methods}

Patients. The present study was approved by the medical ethics committee of Ehime University Hospital (Tōon, Japan) for the Protection of Human Subjects. Informed written consent was obtained from all patients, and the collection of samples was approved by the Institutional Review Board.

A total of 53 patients with clinically diagnosed T1-2N0 OSCC (4) scheduled for radical resection of their tumor(s) and SNB between September 2006 and June 2013 were eligible to participate in the present study. The primary sites were the tongue, gingiva, oral floor, buccal mucosa and lip in 50, 36, 8,5 and $1 \%$ of tumors, respectively. The histological differentiation of the samples was classified in accordance with the World Health Organization classification (23). The mode of cancer invasion was classified into five grades according to the classification proposed by Yamamoto and Kohama (YK classification): YK-1, well-defined border; YK-2, cords, less marked border; YK-3, groups of cells, no distinct border; YK-4C, diffuse invasion, cord-like type; and YK4-D, diffuse invasion, widespread type (24).

Serum collection and IL-6 ELISA. Prior to surgery, serum was collected and immediately frozen at $-80^{\circ} \mathrm{C}$ until being used for the IL-6 assay. Serum IL-6 levels were analyzed using a human IL-6 ELISA kit (cat. no. S6056; BioLegend, Inc., San Diego, CA, USA) according to the manufacturer's protocol. The cut-off value of $20.0 \mathrm{pg} / \mathrm{ml}$ was selected based on the receiver operating characteristic (ROC) curve.

SNB. SNB has received considerable attention for its role in the decision of whether to perform neck dissection (11). The day prior to surgery, a total of $0.4 \mathrm{ml}(74 \mathrm{MBq}){ }^{99 \mathrm{~m}} \mathrm{Tc}-\mathrm{tin}$ colloid was slowly injected around the primary tumor at four points. The sentinel lymph node (SN) was detected by scintigraphy $2 \mathrm{~h}$ following this. The middle and lower portions of the face were covered with a lead plate to reduce shine-through, and four Eppendorf mini tubes, each containing $30 \mu \mathrm{l}$ radioisotope solution, were placed at the chin, the mandibular angle and the anterior and posterior ends of the clavicle to serve as point markers. A small incision was made in the skin above the $\mathrm{SN}$ at operation. Radioactivity of the $\mathrm{SN}$ was measured with the gamma probe Neo 2000 (Navidea Biopharmaceuticals, Dublin, OH, USA). The SN was rinsed quickly in saline solution to remove attached blood, the peripheral adipose tissue and connective tissue were carefully stripped off, and the SN was subsequently divided in two. One half of each lymph node was examined through sectioning (200 $\mu \mathrm{m}$ thick) of the maximal cut surface of the specimen, stained with hematoxylin and eosin and diagnosed as metastatic or non-metastatic. The remaining half of the lymph node was analyzed by one step nucleic acid amplification (OSNA) to calculate the cytokeratin-19 (CK-19) mRNA copy number as previously described (25). A previous study
Table I. Patient and tumor characteristics.

\begin{tabular}{|c|c|}
\hline Clinicopathological characteristic & Number $(\%)$ \\
\hline Number of patients & 53 \\
\hline Age, median (range) & $68.5(40-91)$ \\
\hline \multicolumn{2}{|l|}{ Sex } \\
\hline Male & $31(58.5)$ \\
\hline Female & $22(41.5)$ \\
\hline \multicolumn{2}{|l|}{ WHO (grade) } \\
\hline 1 & $38(71.7)$ \\
\hline 2 & $12(22.6)$ \\
\hline 3 & $3(5.7)$ \\
\hline \multicolumn{2}{|l|}{ Stage } \\
\hline 1 & $24(45.3)$ \\
\hline 2 & $27(50.9)$ \\
\hline 3 & 0 \\
\hline 4 & $2(3.8)$ \\
\hline \multicolumn{2}{|l|}{ SNB } \\
\hline+ & $10(18.9)$ \\
\hline- & $43(81.1)$ \\
\hline Pathological T4 (bone invasion) & $7(13.2)$ \\
\hline \multicolumn{2}{|l|}{ Mode of cancer invasion } \\
\hline YK-1 & $2(3.8)$ \\
\hline YK-2 & $7(13.2)$ \\
\hline YK-3 & $27(50.9)$ \\
\hline YK-4C & $16(30.2)$ \\
\hline YK-4D & $1(1.9)$ \\
\hline \multicolumn{2}{|l|}{ Primary sites } \\
\hline Tongue & $24(45.3)$ \\
\hline Gingiva & $23(43.4)$ \\
\hline Floor of the mouth & $4(7.5)$ \\
\hline Buccal mucosa & $1(1.9)$ \\
\hline Lip & $1(1.9)$ \\
\hline
\end{tabular}

WHO, World Health Organization; SNB, sentinel node biopsy; YK, Yamamoto and Kohama classification.

classified OSNA levels in OSCC as follows: CK19 mRNA copies $<300 / \mu 1$ was designated as negative, and $>300 / \mu 1$ as positive (25). Neck dissection was performed in metastatic cases, determined by either histopathological or genetic examination.

Statistical analysis. Kaplan-Meier curves and log-rank tests were utilized to assess any differences in survival times between the treatment groups. A multivariate analysis was performed to evaluate impact factors on survival using Cox's proportional hazards regression model. Differences between the groups of categorical data were analyzed using two-sided Fisher's exact tests. $\mathrm{P}<0.05$ was considered to indicate a statistically significant difference. GraphPad Prism statistical software version 5 (GraphPad Software, Inc., La Jolla, CA, USA) was used for statistical analysis. 
Table II. Summary of SNB results.

\begin{tabular}{|c|c|c|c|c|c|}
\hline Cases & $\mathrm{n}$ & Identification rate $(\%)$ & SN positive rate $(\%)$ & False negative rate $(\%)$ & Accuracy $(\%)$ \\
\hline All & 53 & $100(53 / 53)$ & $18.9(10 / 53)$ & $28.6(4 / 14)$ & $92.5(49 / 53)$ \\
\hline pT1-2 & 46 & $100(46 / 46)$ & $13(6 / 46)$ & $40(4 / 10)$ & $91.3(42 / 46)$ \\
\hline pT3-4 & 7 & $100(7 / 7)$ & $57.1(4 / 7)$ & $0(0 / 4)$ & $100(7 / 7)$ \\
\hline
\end{tabular}

SNB, sentinel node biopsy; p, pathological stage.

A

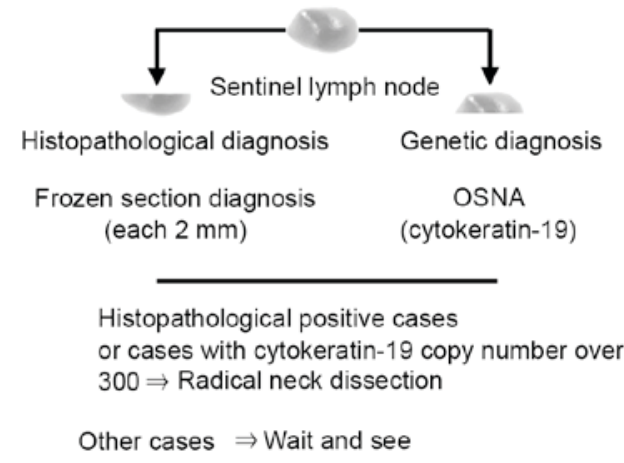

B

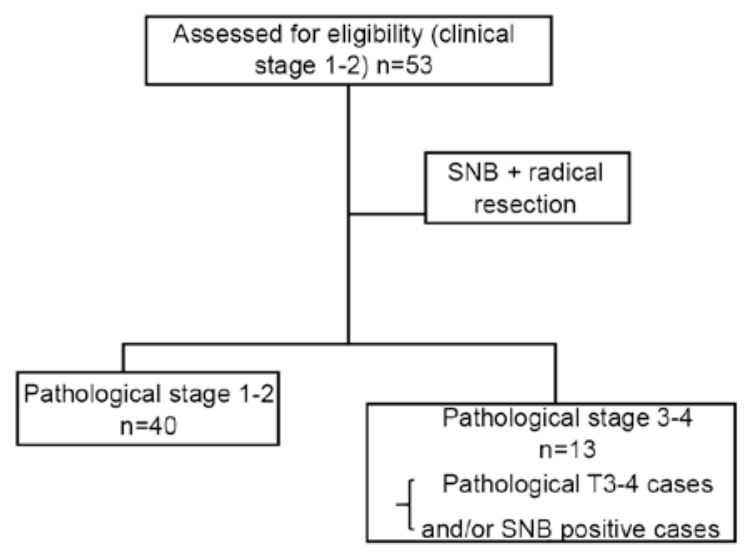

Figure 1. (A) SNB procedure and (B) diagnosis of pathological stage. SNB, sentinel node biopsy; OSNA, one step nucleic acid amplification.

\section{Results}

SNB. Table I lists the patient and tumor characteristics. A total of 53 patients with clinically diagnosed T1-2N0 OSCC undergoing SNB were included in the present study. There were 31 male and 22 female patients, with a mean age of 69 years (range, 40-91 years). The mean number of SNs identified was 1.9 (range, 1-5). In total, 19 patients had $1 \mathrm{SN}$ removed, 22 patients had 2 SNs, 11 patients had 3 SNs and 1 patient had 5 SNs removed. The SNB results are summarized in Fig. 1 and Table II. The SN metastases were pathologically and genetically diagnosed by the SNB specimens in $18.9 \%$ of cases (10/53 cases). The identification rate by SNB was $100 \%$ (53/53 cases), accuracy was $92.5 \%$ (49/53 cases), the SN positive rate was $18.9 \%(10 / 53)$ and the false negative rate was $28.6 \%$ (4/14 cases). In 46 patients with pathological T1-2, the identification rate by SNB was $100 \%$ (46/46 cases), accuracy was $90.3 \%$ (42/46 cases), SN positive rate was $13 \%(6 / 46)$ and the false negative rate was $29 \%$ (1-sensitivity). In patients with pathological T3-4, the identification rate of SNB was $100 \%$ (7/7 cases), the accuracy was $100 \%$ (7/7 cases), the SN positive rate was $57.1 \%$ (4/7) and the false negative rate was $0 \%$. Of these 53 patients, $13(24.5 \%)$ were excluded from the pathological stage 1-2 group due to presence of pathological T 3-4 stage (including bone invasion) tumor and $\mathrm{SN}$ metastasis (Tables II and III).

Association between the SNB status of patients with OSCC and serum IL-6 levels. The cut-off value of $20.0 \mathrm{pg} / \mathrm{ml}$ was selected based on the ROC curve. ELISA was performed for IL-6 in the 53 patients exhibiting cT1-T2 and evaluated for association with lymph node metastasis. Analysis revealed no association between SN metastasis and IL-6 level ( $\mathrm{P}=0.73$; Table IV).

Kaplan-Meier survival plots comparing SNB and serum IL-6 levels. Kaplan-Meier survival plots comparing the overall survival (OS) and disease-free survival (DFS) fractions of each group are depicted in Fig. 2. OS ( $\log$ rank $\mathrm{P}=0.03)$ and DFS (Log rank $\mathrm{P}=0.02$ ) were significantly lower among the patients whose SNB status and tumor bone invasion status were positive (Fig. 2A and B). Among the 53 patients, the 24 patients of the low serum IL-6 group tended to survive longer ( $\mathrm{P}=0.33$ in $\mathrm{OS}, \mathrm{P}=0.047$ in DFS), but the difference was not significant for OS (Fig. 2C and D). A total of 13 patients were diagnosed with lymph node metastasis by SNB or were reclassified to pathological stage $\mathrm{T} 4$ from evaluation of the surgically resected specimen. Thus, 40 patients were strictly defined as pathological stage 1-2 OSCC. Among them, the OS of patients with low serum IL-6 levels was not statistically significant different compared with those with high serum levels ( $\mathrm{P}=0.24$; Fig. $2 \mathrm{E})$ but DFS of the patients with low serum IL-6 levels was significantly longer compared with those with high serum IL-6 $(\mathrm{P}=0.012$; Fig. $2 \mathrm{~F})$. For patients with pathological stage 1-2 tumors strictly defined by SNB and pathological examination, OS and DFS were $100 \%$ in the low serum IL-6 group in the follow-up period (Fig. 2E and F).

\section{Discussion}

The present study demonstrated that staging by SNB and preoperative serum IL-6 level exhibited a high prognostic value 
Table III. Metastasis in SNB positive and negative cases.

\begin{tabular}{lccc}
\hline Cases & Metastasis (+) & Metastasis (-) & Total \\
\hline All & 14 & 39 & 53 \\
SNB (+) & 10 & 0 & 10 \\
SNB (-) & 4 & 39 & 43 \\
pT1-2 & 10 & 36 & 46 \\
SNB (+) & 6 & 0 & 6 \\
SNB (-) & 4 & 36 & 40 \\
pT3-4 & 4 & 3 & 7 \\
SNB (+) & 4 & 0 & 4 \\
SNB (-) & 0 & 3 & 3 \\
\hline
\end{tabular}

SNB, sentinel node biopsy; p, pathological stage.

Table IV. Serum IL-6 levels in SNB negative and positive cases.

\begin{tabular}{lcc}
\hline Serum IL-6 & SNB negative & SNB positive \\
\hline High & 25 & 5 \\
Low & 18 & 5 \\
\hline
\end{tabular}

SNB, sentinel node biopsy; IL-6, interleukin-6.

in patients with OSCC. In patients with early-stage OSCC, the nodal status of the cervical lymph nodes remained an important prognostic factor. Advances in imaging technology have made additional techniques available, including US, CT, MRI and PET imaging. However, the diagnostic ability of US, CT and MRI is primarily based on node morphology and size criteria, with nodes $<10 \mathrm{~mm}$ generally considered to not harbour metastasis. Attempts with functional imaging using PET scanning were also revealed not to be efficient in detecting occult cervical metastasis. Since the early 1980 s, treatment of patients with clinical NO OSCC has evolved from observation to elective neck dissection for all but the earliest-stage types of cancer of the oral cavity, with elective neck dissection becoming the standard of care at the majority of institutions treating a large number of patients with cancer in the 1990s (7). However, elective neck dissection is a surgical procedure that was revealed to be overtreatment for $\sim 70 \%$ of patients with $\mathrm{cN}$, who were revealed to have a pathological node-negative neck (8). Observation was the primary therapeutic modality employed in the management of patients with N0 neck tumors, yet it soon became evident that $\sim 30 \%$ of observed patients developed cervical node metastasis despite tumor control at the primary site (26-28).

An SN is the first lymph node(s) to which cancer cells are most likely to spread from a primary tumor. SNB may be used to help determine the extent of the disease, and to stage and guide the use of adjuvant radiation and chemotherapy to reduce disease recurrence. As SNB involves less extensive surgery and the removal of fewer lymph nodes compared with neck dissection, the risk of adverse effects is lower. In
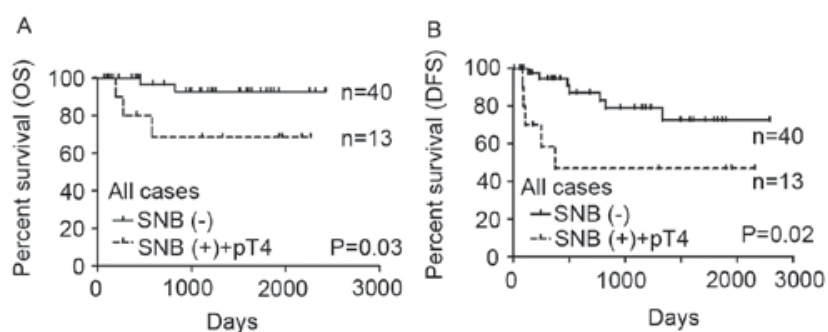

$\mathrm{C}$

D
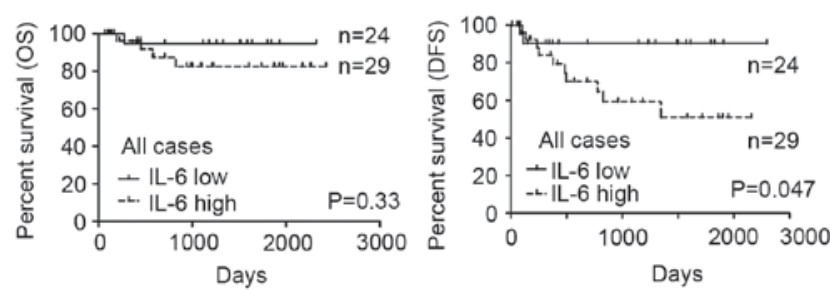

E

$\mathrm{F}$
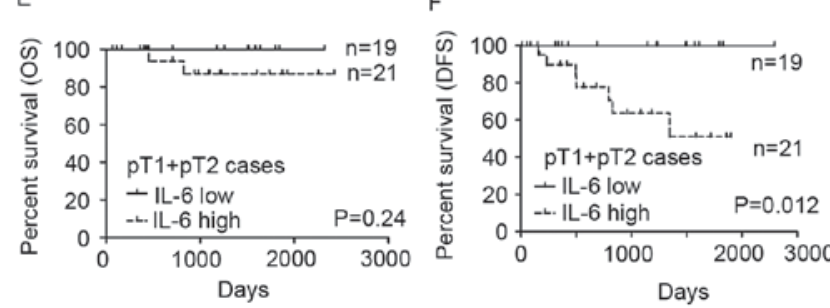

Figure 2. Kaplan-Meier survival plots comparing (A) SNB and OS, (B) SNB and DFS, (C) serum IL-6 levels and OS, (D) serum IL-6 levels and DFS in all cases, and (E) serum IL-6 levels and OS and (F) serum IL-6 levels and DFS in patients with pT1-2 oral squamous cell carcinoma. SNB, sentinel node biopsy; OS, overall survival; DFS, disease-free survival; IL-6, interleukin-6; $\mathrm{p}$, pathological stage.

the present study, the SNB positive rate was $18.9 \%(10 / 53)$, but the delayed neck metastasis was $7.5 \%$ (4/53). Consistent with previous reports of cervical metastasis, the present study revealed a lymph node metastasis rate of $26.4 \%$.

The present results suggested that SNB may improve the prognosis of patients with OSCC, with a significantly higher OS $(\mathrm{P}=0.03)$ and DFS $(\mathrm{P}=0.02)$ in patients whose $\mathrm{SNB}$ status was negative. However, a few cases of delayed neck metastasis and local recurrence were observed, even in SNB negative cases. A possible cause may be the lack of rapid and accurate intraoperative detection of metastatic disease in the $\mathrm{SN}(\mathrm{s})(29,30)$. In addition, the occurrence of a second primary tumor is $3-7 \%$ higher per year in patients with OSCC compared with other malignancies (31). Therefore, the identification of suitable and reliable biomarkers is essential for achieving early detection and treatment, which may reduce mortality rates in patients with OSCC. However, several serum cytokines were previously examined in patients with OSCC by using a multiplexed measurement system, revealing that the serum IL-6 level tended to negatively correlate with favorable outcome in these patients, with OS and DFS rates of $100 \%$ in patients with early-stage OSCC diagnosed by SNB with low levels of serum IL-6. Furthermore, adjuvant and/or neo-adjuvant therapies may be administered to patients with high levels of serum IL-6, despite having only early-stage OSCC. In this previous study, there were 4 false negative cases (delayed neck metastasis). It was impossible to analyze the statistical significance with respect to correlation between the serum IL-6 level and frequency of the delayed neck metastasis in SNB 
negative cases as there were few applicable cases. Therefore, this requires additional investigation.

Previous studies have revealed that stromal fibroblasts isolated from various types of cancer produced a significant amount of IL-6, which induced tumor proliferation, invasion, migration and angiogenesis $(19,32,33)$. An on-going prospective study may further elucidate the prognostic and predictive significance of IL-6, which may warrant future clinical trials of an IL-6 inhibitor for patients with high-risk OSCC.

In conclusion, the present study revealed that SNB staging and preoperative serum IL-6 level have a high prognostic value in patients with OSCC, and IL-6 may be associated with poor clinical outcome. Future studies are required to increase understanding of the biological significance underlying the association of IL-6 with OSCC and the tumor microenvironment, including how these findings may be combined with other treatment approaches, including radiation and chemotherapy, which may contribute to the continued improvement of OSCC treatments.

\section{Acknowledgements}

The present study was supported by a Grant-in-Aid for Scientific Research from the Ministry of Education, Culture, Sports, Science and Technology of Japan.

\section{References}

1. Jemal A, Siegel R, Ward E, Hao Y, Xu J and Thun MJ: Cancer Statistics, 2009. CA Cancer J Clin 59: 225-249, 2009.

2. Jemal A, Bray F, Center MM, Ferlay J, Ward E and Forman D: Global Cancer Statistics. CA Cancer J Clin 61: 69-90, 2011.

3. Forastiere AA, Goepfert H, Maor M, Pajak TF, Weber R, Morrison W, Glisson B, Trotti A, Ridge JA, Chao C, et al: Concurrent chemotherapy and radiotherapy for organ preservation in advanced laryngeal cancer. N Engl J Med 349: 2091-2098, 2003

4. James DB, Mary KG and Christian W (eds): UICC: TNM classification of malignant tumors. 8th edition. Wiley-Blackwell, Hoboken, NJ, pp272, 2016.

5. Kunishi M, Kayada Y and Yoshiga K: Down-regulated expression of CD44 variant 6 in oral squamous cell carcinomas and its relationship to regional lymph node metastasis. Int J Oral Maxillofac Surg 26: 280-283, 1997.

6. Okada Y, Mataga I, Katagiri M and Ishii K: An analysis of cervical lymph nodes metastasis in oral squamous cell carcinoma. Relationship between grade of histopathological malignancy and lymph nodes metastasis. Int J Oral Maxillofac Surg 32: 284-288, 2003.

7. Myers EN and Fagan J: Treatment of the $\mathrm{N}+$ neck in squamous cell carcinoma of the upper aerodigestive tract. Otolaryngol Clin North Am 31: 671-686, 1998.

8. Stuckensen T, Kovács AF, Adams S and Baum RP: Staging of the neck in patients with oral cavity squamous cell carcinomas: a prospective comparison of PET, ultrasound, CT and MRI. J Craniomaxillofac Surg 28: 319-324, 2000.

9. Cunningham MJ, Johnson JT, Myers EN, Schramm VL Jr and Thearle PB: Cervical lymph node metastasis after local excision of early squamous cell carcinoma of the oral cavity. Am J Surg 152: 361-366, 1986.

10. Broglie MA, Haerle SK, Huber GF, Haile SR and Stoeckli SJ: Occult metastases detected by sentinel node biopsy in patients with early oral and oropharyngeal squamous cell carcinomas: impact on survival. Head Neck 35: 660-666, 2013.

11. Almadori G, Bussu F and Paludetti G: Should there be more molecular staging of head and neck cancer to improve the choice of treatments and thereby improve survival? Curr Opin Otolaryngol Head Neck Surg 16: 117-126, 2008.

12. Woolgar JA and Hall GL: Determinants of outcome following surgery for oral squamous cell carcinoma. Future Oncol 5: 51-61, 2009.
13. Kreppel M, Drebber U, Rothamel D, Eich HT, Kübler A, Scheer M and Zöller JE: Prognostic impact of different TNM-based stage groupings for oral squamous cell carcinoma. Head Neck 33: 1467-1475, 2011.

14. Biancotto A, Feng X, Langweiler M, Young NS and McCoy JP Effect of anticoagulants on multiplexed measurement of cytokine/chemokines in healthy subjects. Cytokine 60: 438-446, 2012.

15. Kishimoto T, Akira S, Narazaki $M$ and Taga T: Interleukin-6 family of cytokines and gp130. Blood 86: 1243-1254, 1995.

16. Shinriki S, Jono H, Ota K, Ueda M, Kudo M, Ota T, Oike Y, Endo M, Ibusuki M, Hiraki A, et al: Humanized anti-interleukin-6 receptor antibody suppresses tumor angiogenesis and in vivo growth of human oral squamous cell carcinoma. Clin Cancer Res 15: 5426-5434, 2009

17. Liu Q, Li G, Li R, Shen J, He Q, Deng L, Zhang C and Zhang J: IL-6 promotion of glioblastoma cell invasion and angiogenesis in U251 and T98 G cell lines. J Neurooncol 100: 165-176, 2010.

18. Shinriki S, Jono H, Ueda M, Ota K, Ota T, Sueyoshi T, Oike Y, Ibusuki M, Hiraki A, Nakayama $\mathrm{H}$, et al: Interleukin-6 signalling regulates vascular endothelial grow th factor-C synthesis and lymphangiogenesis in human oral squamous cell carcinoma. J Pathol 225: 142-150, 2011.

19. Nagasaki T, Hara M, Nakanishi H, Takahashi H, Sato $M$ and Takeyama H: Interleukin- 6 released by colon cancer-associated fibroblasts is critical for tumour angiogenesis: Anti-interleukin-6 receptor antibody suppressed angiogenesis and inhibited tumour-stroma interaction. Br J Cancer 110: 469-478, 2014.

20. Chen Z, Malhotra PS, Thomas GR, Ondrey FG, Duffey DC, Smith CW, Enamorado I, Yeh NT, Kroog GS, Rudy S, et al: Expression of proinflammatory and proangiogenic cytokines in patients with head and neck cancer. Clin Cancer Res 5: 1369-1379, 1999.

21. Duffy SA, Taylor JM, Terrell JE, Islam M, Li Y, Fowler KE, Wolf GT and Teknos TN: Interleukin-6 predicts recurrence and survival among head and neck cancer patients. Cancer 113: 750-757, 2008.

22. Chang KP, Kao HK, Wu CC, Fang KH, Chang YL, Huang YC, Liu SC and Cheng MH: Pretreatment interleukin-6 serum levels are associated with patient survival for oral cavity squamous cell carcinoma. Otolaryngol Head Neck Surg 148: 786-791, 2013.

23. Bosman FT, Carneiro F, Hruban RH and Theise ND: WHO Classification of tumours of the digestive system, fourth edition. WHO Classification of Tumours 3: 417, 2010.

24. Yamamoto E, Kohama G, Sunakawa H, Iwai M and Hiratsuka H: Mode of invasion, bleomycin sensitivity, and clinical course in squamous cell carcinoma of the oral cavity. Cancer 51: 2175-2180, 1983.

25. Goda H, Nakashiro K, Oka R, Tanaka H, Wakisaka H, Hato N, Hyodo $\mathrm{M}$ and Hamakawa $\mathrm{H}$ : One-step nucleic acid amplification for detecting lymph node metastasis of head and neck squamous cell carcinoma. Oral Oncol 48: 958-963, 2012.

26. Whitehurst JO and Droulias CA: Surgical treatment of squamous cell carcinoma of the oral tongue: Factors influencing survival. Arch Otolaryngol 103: 212-215, 1977.

27. Yuen AP, Wei WI, Wong YM and Tang KC: Elective neck dissection versus observation in the treatment of early oral tongue carcinoma. Head Neck 19: 583-588, 1997.

28. Kowalski LP: Results of salvage treatment of the neck in patients with oral cancer. Arch Otolaryngol Head Neck Surg 128: 58-62, 2002.

29. Iii RPZ, Todd DW, Renner GJ and Singh A: Intraoperative radiolymphoscintigraphy for detection of occult nodal metastasis in patients with head and neck squamous cell carcinoma. Otolaryngol Head Neck Surg 122: 662-666

30. Hyde NC, Prvulovich E, Newman L, Waddington WA, Visvikis D and Ell P: A new approach to pre-treatment assessment of the N0 neck in oral squamous cell carcinoma: The role of sentinel node biopsy and positron emission tomography. Oral Oncol 39: 350-360, 2003

31. Day GL and Blot WJ: Second primary tumors in patients with oral cancer. Cancer 70: 14-19, 1992.

32. Al-Rakan MA, Colak D, Hendrayani SF, Al-Bakheet A, Al-Mohanna FH, Kaya N, Al-Malik O and Aboussekhra A: Breast stromal fibroblasts from histologically normal surgical margins are pro-carcinogenic. J Pathol 231: 457-465, 2013.

33. Chen SX, Xu XE, Wang XQ, Cui SJ, Xu LL, Jiang YH, Zhang Y, Yan HB, Zhang Q, Qiao J, et al: Identification of colonic fibroblast secretomes reveals secretory factors regulating colon cancer cell proliferation. J Proteomics 110: 155-171, 2014. 